\title{
Type of featural attention differentially modulates hMT+ responses to illusory motion aftereffects
}

Citation for published version (APA):

Castelo-Branco, M., Kozak, L. R., Formisano, E., Teixeira, J., Xavier, J., \& Goebel, R. (2009). Type of featural attention differentially modulates hMT+ responses to illusory motion aftereffects. Journal of Neurophysiology, 102(5), 3016-3025. https://doi.org/10.1152/jn.90812.2008

Document status and date:

Published: 01/01/2009

DOI:

10.1152/jn.90812.2008

Document Version:

Publisher's PDF, also known as Version of record

Document license:

Taverne

\section{Please check the document version of this publication:}

- A submitted manuscript is the version of the article upon submission and before peer-review. There can be important differences between the submitted version and the official published version of record.

People interested in the research are advised to contact the author for the final version of the publication, or visit the DOI to the publisher's website.

- The final author version and the galley proof are versions of the publication after peer review.

- The final published version features the final layout of the paper including the volume, issue and page numbers.

Link to publication

\footnotetext{
General rights rights.

- You may freely distribute the URL identifying the publication in the public portal. please follow below link for the End User Agreement:

www.umlib.nl/taverne-license

Take down policy

If you believe that this document breaches copyright please contact us at:

repository@maastrichtuniversity.nl

providing details and we will investigate your claim.
}

Copyright and moral rights for the publications made accessible in the public portal are retained by the authors and/or other copyright owners and it is a condition of accessing publications that users recognise and abide by the legal requirements associated with these

- Users may download and print one copy of any publication from the public portal for the purpose of private study or research.

- You may not further distribute the material or use it for any profit-making activity or commercial gain

If the publication is distributed under the terms of Article $25 \mathrm{fa}$ of the Dutch Copyright Act, indicated by the "Taverne" license above, 


\title{
Type of Featural Attention Differentially Modulates $\mathrm{hMT}^{+}$Responses to Illusory Motion Aftereffects
}

\author{
Miguel Castelo-Branco, ${ }^{1, *}$ Lajos R. Kozak, ${ }^{1-3, *}$ Elia Formisano, ${ }^{4}$ João Teixeira, ${ }^{5}$ João Xavier, ${ }^{5}$ \\ and Rainer Goebel ${ }^{\mathbf{4}}$ \\ ${ }^{1}$ Visual Neuroscience Laboratory, IBILI, Faculty of Medicine, University of Coimbra, Coimbra, Portugal; ${ }^{2}$ Institute for Psychology, \\ Hungarian Academy of Sciences, Budapest, Hungary; ${ }^{3}$ MR Research Center, Szentagothai Knowledge Center, Semmelweis University, \\ Budapest, Hungary; ${ }^{4}$ Faculty of Psychology, Universiteit Maastricht, Maastricht, The Netherlands; and ${ }^{5}$ Department of Neuroradiology, \\ Hospital Geral de Santo Antonio, Porto, Portugal
}

Submitted 26 July 2009; accepted in final form 21 August 2009

\begin{abstract}
Castelo-Branco M, Kozak LR, Formisano E, Teixeira J, Xavier J, Goebel R. Type of featural attention differentially modulates $\mathrm{hMT}^{+}$responses to illusory motion aftereffects. J Neurophysiol 102: 3016-3025, 2009. First published August 26, 2009; doi:10.1152/jn.90812.2008. Activity in the human motion complex $\left(\mathrm{hMT}^{+} / \mathrm{V} 5\right)$ is related to the perception of motion, be it either real surface motion or an illusion of motion such as apparent motion (AM) or motion aftereffect (MAE). It is a longlasting debate whether illusory motion-related activations in $\mathrm{hMT}^{+}$ represent the motion itself or attention to it. We have asked whether $\mathrm{hMT}^{+}$responses to MAEs are present when shifts in arousal are suppressed and attention is focused on concurrent motion versus nonmotion features. Significant enhancement of $\mathrm{hMT}^{+}$activity was observed during MAEs when attention was focused either on concurrent spatial angle or color features. This observation was confirmed by direct comparison of adapting (MAE inducing) versus nonadapting conditions. In contrast, this effect was diminished when subjects had to report on concomitant speed changes of superimposed AM. The same finding was observed for concomitant orthogonal real motion (RM), suggesting that selective attention to concurrent illusory or real motion was interfering with the saliency of MAE signals in $\mathrm{hMT}^{+}$. We conclude that MAE-related changes in the global activity of $\mathrm{hMT}^{+}$are present provided selective attention is not focused on an interfering feature such as concurrent motion. Accordingly, there is a genuine MAE-related motion signal in $\mathrm{hMT}^{+}$that is neither explained by shifts in arousal nor by selective attention.
\end{abstract}

\section{N T R O D U C T I O N}

The motion aftereffect is an illusion of motion perceived on static test patterns after prolonged directional motion adaptation (Anstis et al. 1998; Verstraten et al. 1998) generally in the opposite direction to the adapting movement. The underlying neuronal process is generally considered to be an adaptationinduced imbalance in the activation of directional-selective neurons in human motion complex (area $\left.\mathrm{hMT}^{+} / \mathrm{V} 5\right)$. It is well known that modulation of activity in $\mathrm{hMT}^{+}$is related to the perception of global motion (Braddick et al. 2000; CasteloBranco et al. 2002, 2006; Goebel et al. 1998; Huk and Heeger 2002; Sunaert et al. 1999; Tootell and Taylor 1995; Watson et al. 1993) and that response levels also depend on attentional modulation (Beauchamp et al. 1997; Buchel et al. 1998; Kastner et al. 1999; O'Craven et al. 1997). However, it is still an open question whether net blood-oxygenation-level-dependent

\footnotetext{
* M. Castelo-Branco and L. R. Kozak contributed equally to this work.

Address for reprint requests and other correspondence: M. Castelo-Branco, Visual Neuroscience Laboratory, IBILI-Faculdade de Medicina, Az. de Sta Comba Celas, 3000-354 Coimbra, Portugal (E-mail: mcbranco@ibili.uc.pt).
}

(BOLD) responses in area $\mathrm{hMT}^{+}$to motion aftereffects (MAE) (Corbetta et al. 1990; Culham et al. 1999; Hautzel et al. 2001; He et al. 1998; Taylor et al. 2000; Tootell et al. 1995) reflect only nonspecific shifts in arousal and/or specific attentional modulation of activity instead of global motion adaptationrelated responses (Huk et al. 2001).

It is worth noting in this context that even weak motion signals can be modulated by selective attention (Alais and Blake 1999; Seidemann and Newsome 1999; Treue and Martinez Trujillo 1999) or contextual influences (Kozak and Castelo-Branco 2009). Huk et al. in 2001 observed no net MAE-related increase of $\mathrm{hMT}^{+}$activity in a task directed to near threshold stimulus motion and interpreted this result as an indication that the observed BOLD response characteristics were explained by shifts in attention and/or nonspecific effects of arousal. However, selective attention to concurrent motion may be confounded by interference due to the presence of dual motion processing. Generalization to other selective attention conditions that require focusing on motion unrelated features is also important. In other words, the presence of a net MAE-related signal may require selective attention to features that do not compete for motion processing. This can be achieved by controlling attention using tasks either with concurrent motion or motion-unrelated stimuli. If modulation of $\mathrm{hMT}^{+}$activity during perception of MAEs is specifically present in the motion-unrelated attention tasks, then the presence of a net MAE signal in hMT + requires the absence of concomitant processing of competing motion cues. This would render the interesting (and replicable) results of Huk et al. (2001) not generalizable for concurrent attention to nonmotion features.

In contrast with Huk et al. (2001), we have therefore added in our experimental approach concurrent illusory motion distinct from MAE in addition to real surface motion (RM). A good candidate was apparent motion (AM), an illusion of movement that can be induced when spatially segregated visual stimuli are presented in alternation: subjects can then perceive a visual stimulus smoothly traversing the intervening space where no physical stimulus exists. It has already been shown that the human motion-sensitive complex is the first within the dorsal processing stream to respond with a clear increase in signal intensity to AM stimuli (Goebel et al. 1998; Liu et al. 2004; Mikami et al. 1986a,b; Muckli et al. 2002; Newsome et al. 1986).

We conducted a series of experiments for the assessment of interactions among superimposed motion-unrelated, AM, RM, 
and MAE signals in $\mathrm{hMT}^{+}$. Our prediction was that $\mathrm{hMT}^{+}$ sensitivity to MAE can be differentially modulated by selective featural attention, depending on whether concurrent features to be attended are motion related or not. We could confirm this prediction by using distinct featural attention tasks, thereby reconciling the apparently contradictory results of Tootell et al. (1995) and Huk et al. (2001).

\section{METHODS}

\section{Data acquisition}

Data were acquired for a total of 20 subjects. Experiments $1 a$ and $1 b$ were performed with four and eight subjects at 1.5 and 3 Tesla, respectively, and experiments $2 a$ and $2 b$ with four and eight subjects at 1.5 and 3 Tesla, respectively.

EXPERIMENT 1A. This first set of functional magnetic resonance imaging (fMRI) experiments was performed in four subjects at $1.5 \mathrm{~T}$ (Philips ACS-NT; Philips, Eindhoven, The Netherlands) by using the standard head coil and a gradient echo echo-planar imaging (EPI) sequence $\left(\mathrm{TR}=2,083 \mathrm{~ms}, \mathrm{TE}=40 \mathrm{~ms}, \mathrm{FOV}=224 \times 224 \mathrm{~mm}^{2}, 22\right.$ slices, voxel size: $3.5 \times 3.5 \times 5 \mathrm{~mm}^{3}$ ). A T1-weighted threedimensional (3D) magnetization prepared rapid acquisition gradient echo or fast field echo scan was recorded in the same session as the functional measurements (voxel size: $1.0 \times 1.0 \times 1.0 \mathrm{~mm}^{3}$ ).

EXPERIMENT $1 B$. This second set of fMRI experiments was performed in eight subjects at $3 \mathrm{~T}$ (Achieva, Philips Medical System). BOLD-sensitive T2*-weighted echo-planar imaging sequences (TR $=$ $2,000 \mathrm{~ms}, \mathrm{TE}=30 \mathrm{~ms}, \mathrm{FOV}=240 \times 240 \mathrm{~mm}^{2}$ ) were obtained with 3 -mm slice $(n=36)$ thickness with $3 \mathrm{~mm} \times 3 \mathrm{~mm}$ in-plane resolution. High-resolution anatomical images were acquired for each subject using a T1-weighted 3D TFE sequence yielding images with a $1 \times 1 \times 1 \mathrm{~mm}$ resolution.

EXPERIMENT 2A. This third set of functional imaging experiments was performed in four participants (only 1 of which had participated in experiment 1) at $1.5 \mathrm{~T}$ in a Philips Gyroscan Intera (Philips) scanner by using the standard head coil and a gradient echo EPI sequence $\left(\mathrm{TR}=3,000 \mathrm{~ms}, \mathrm{TE}=50 \mathrm{~ms}, \mathrm{FOV}=224 \times 224 \mathrm{~mm}^{2}, 30\right.$ slices, voxel size: $3.5 \times 3.5 \times 4 \mathrm{~mm}^{3}$ ) A T1-weighted $3 \mathrm{D}$ magnetization prepared rapid acquisition gradient echo scan was recorded in the same session as the functional measurements (voxel size: $1.0 \times 1.0 \times$ $\left.1.0 \mathrm{~mm}^{3}\right)$.

EXPERIMENT 2B. This final set of functional imaging experiments was performed in eight participants (only 2 of which had participated in experiment 1 and none in experiment $2 a$ ) at $3 \mathrm{~T}$ in a Siemens Allegra scanner (Siemens AG, Erlangen, Germany) by using the standard head coil and a gradient echo EPI sequence (TR $=2,000 \mathrm{~ms}$, $\mathrm{TE}=30 \mathrm{~ms}$, effective FOV $=190 \times 190 \mathrm{~mm}^{2}$, 33 slices, voxel size: $2.97 \times 2.97 \times 3 \mathrm{~mm}^{3}$ ) A T1-weighted 3D Tfl.ADNI anatomical sequence was recorded in the same session as the functional measurements (voxel size: $1.0 \times 1.0 \times 1.0 \mathrm{~mm}^{3}$ ).

\section{Visual stimuli and paradigms}

We have chosen plaid stimuli (see Figs. 1 and 2) because they robustly activate $\mathrm{hMT}^{+}$and induce strong MAEs.

EXPERIMENT 1A. Plaid stimuli (see Fig. 1) were constructed by superimposing square-wave gratings with 0.3 average duty cycle and angles of approximately $\pm 75^{\circ}$ in a $12^{\circ}$ circular aperture. We have used plaids with opposite contrast polarity of dark and light stripes $\left(\sim 12\right.$ and $\left.36 \mathrm{~cd} \cdot \mathrm{m}^{-2}\right)$ on intermediate background luminance $(\sim 24$ $\left.\mathrm{cd} \cdot \mathrm{m}^{-2}\right)$. Typical intersection luminances were 10 or $30 \mathrm{~cd} \cdot \mathrm{m}^{-2}$. Plaid velocities were either 8 or $16^{\circ} \cdot \mathrm{s}^{-1}$. Subjects viewed upward moving plaids ( 8 volumes, $16 \mathrm{~s}$ ) separated by rest conditions. Any given rest condition was either a fixation baseline ( 6 volumes) or a stationary plaid (8 volumes), which evoked a salient unidirectional motion aftereffect. Subjects performed either an angle or a color task. In the angle task, subjects had to report whether the angle of the fixation cross (changing every $2 \mathrm{~s}$ ) was larger or smaller than the plaid angle (see Fig. 1). This task required direct stable attention to plaid stimuli, but the subjects still had to report on a motion-unrelated

\section{Fixation}

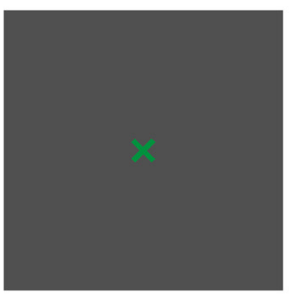

\section{Angle Task Cue}

\section{or}

\section{Color Task Cue}

Moving
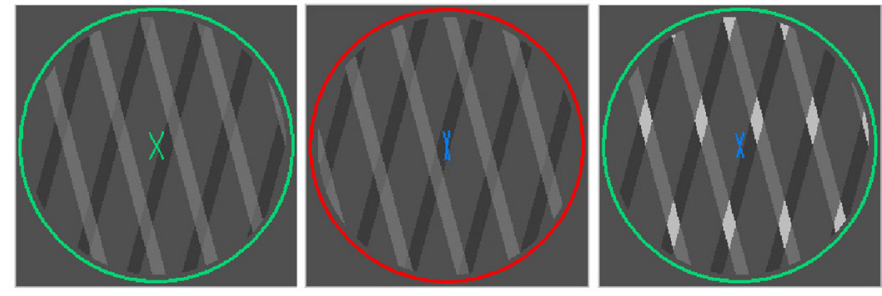

\section{Moving}



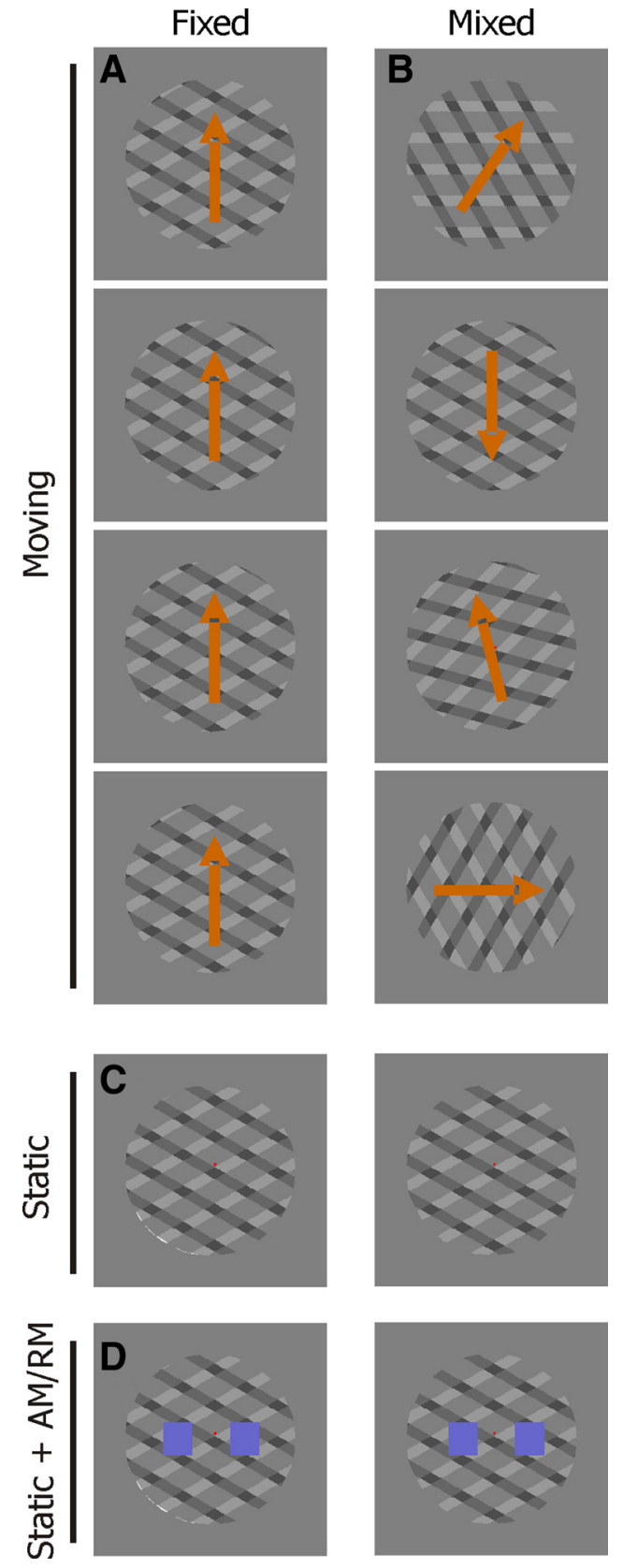

FIG. 2. Experimental design for experiments $2 a$ and $2 b(n=4$ and $n=8$, respectively). Sixteen-second blocks of plaid motion and static plaids were combined and presented in a block design manner in these experiments. Motion blocks had either constant direction of motion (fixed; $A$ ) or the direction changed in every $2 \mathrm{~s}(\operatorname{mix} e d ; B)$. There were motion periods with and without overlaid apparent motion (only in experiment $2 a$ ). Static blocks were also presented with $(D)$ and without $(C)$ overlaid apparent (AM) or real motion (RM) tasks (the speed judgment of moving squares RM task was only applied in experiment $2 b$ ). Note that there are no contrast transients between moving and static plaids.

feature (angle between grating stripes). In the color task, stable attention was required on features not directly related to plaid stimuli. In this task, subjects had to report whether the fixation-cross (changing every $2 \mathrm{~s}$ ) had the same or different color from the curve outlining the stimulus aperture (see Fig. 1). Each task was repeated in eight blocks per run interleaved in a balanced manner (concerning plaid luminance types-see Supplementary Fig. $\mathrm{S}^{1}$ ) across the two exper-

\footnotetext{
${ }^{1}$ The online version of this article contains supplemental data.
}

imental runs. Each block started with a fixation condition, followed by moving plaids, static plaids, moving plaids, and a fixation condition again (that started a new cycle). The shape of the fixation stimulus (cross or circle) cued whether subjects had to perform the color or the angle comparison task during the following motion and static period because there was no difference in the moving and static plaid displays between the two attentional task conditions. Tasks were performed continuously, both during inspection of moving and stationary stimuli, to ensure stable control of selective attention, and to avoid confounding task-related contrasts.

Flow-field experiments were performed to localize motion-sensitive areas in single subjects (in addition to plaid motion contrast). During these measurements, two stationary stimuli (fixation cross and stationary dot pattern, repeated 4 and 10 times) and a motion condition (flow fields, $30^{\circ}$ wide by $23^{\circ}$ high, 400 dots, size $0.06 \times 0.06^{\circ}$, velocity $3.6-14.4 \%$, repeated 5 times for $16 \mathrm{~s}$ ) were alternated.

EXPERIMENT 1B. This experiment was an independent replication of experiment $1 a$, with the additional inclusion of a nonadapting motion (e.g., not eliciting MAEs) control condition (and, as in all experiments, an independent $\mathrm{hMT}^{+}$localizer experiment). Plaid stimuli characteristics were as in experiment $2 a$ (see following text). Each condition was repeated six times across three runs, using a balanced pseudo-randomization based on latin-squares. The color and angle tasks were as in experiment $1 a$.

EXPERIMENT 2A. Sixteen-second epochs of plaid motion and static plaids were combined and presented in a block design manner (Fig. 2). Motion blocks had either constant direction of motion (fixed conditions) or the direction changed in every $2 \mathrm{~s}$ (mixed conditions). There were motion and static blocks with and without overlaid apparent motion as well as intervening fixation periods (which were used to compute baseline activity). As an attentional task, subjects had to track speed changes of the apparent motion stimulus superimposed on the moving and static plaids. All subjects performed this behavioral task $>80 \%$ accuracy.

Plaid stimuli were constructed by superimposing square-wave gratings with 0.25 average duty cycle and angles of approximately $\pm 60^{\circ}$ (directions $\pm 30^{\circ}$ ) in a $4^{\circ}$ circular aperture with $1.5 \mathrm{cpd}$ spatial resolution. Luminance of the background was $\sim 10 \mathrm{~cd} \cdot \mathrm{m}^{-2}$, for the gratings it was $\sim 7.5$ and $\sim 25 \mathrm{~cd} \cdot \mathrm{m}^{-2}$ with the intersection being $\sim 4$ $\mathrm{cd} \cdot \mathrm{m}^{-2}$. The luminance of the blue AM stimulus was $\sim 4 \mathrm{~cd} \cdot \mathrm{m}^{-2}$. Plaid pattern velocity was $1.54^{\circ} \cdot \mathrm{s}^{-1}$. Epochs of plaid motion $(16 \mathrm{~s})$, static plaids (16 s), and fixation ( $8 \mathrm{~s}$ ) were combined and presented in a block design manner. Data were collected in four runs, containing 32 balanced blocks altogether $(8$ with adapting fixed motion without AM, 8 with adapting fixed motion with AM, 8 with nonadapting mixed motion without AM, 8 with nonadapting mixed motion with AM, each block containing a MAE test period with overlaid AM in half of the cases and a fixation period afterward). This means that four blocks of MAE test periods were included that did contain the AM task and four that did not, which allowed for direct comparison of responses to static plaids with and without MAE perception.

Motion direction changed (only under mixed-motion conditions, see following text) in every $2 \mathrm{~s}$ ( 8 evenly distributed directions in a range of $360^{\circ}$ ) with and without overlaid AM. The overlaid apparent motion stimulus consisted of two squares with $0.4^{\circ}$ size separated by $1.6^{\circ}$ oscillating horizontally back and forth (velocity was constant and the square was moving back and forth), such that they were orthogonal to adapting plaid motion. No stimuli were placed in intervening positions across frames, which ensured that no real motion was present. The speed of AM stimuli changed every $4 \mathrm{~s}$; the range of speed changes was as follows: $3.33,4.44,6.66$, and $13.33^{\circ} \cdot \mathrm{s}^{-1}$.

EXPERIMENT 2B. Stimulus properties were similar to experiment $2 a$, except for the inclusion of a new condition: real motion using similar stimuli as in the AM condition overlaid on top of the MAE period (see Fig. 2). Furthermore, AM and RM stimuli were only superimposed on 
the MAE test period. Accordingly, the experimental paradigm was built from 16-s blocks of fixed- or mixed-direction moving plaid and static plaid displays with and without an overlaid apparent or real motion task after the motion adaptation period (Fig. 2). Data were collected in two runs, containing 18 balanced blocks ( 9 with adapting fixed motion and 9 with nonadapting mixed motion, and each containing a fixation period, adapting period and MAE test period with or without AM/RM superimposed during the MAE period). Subjects had to track speed changes of the apparent motion or real motion stimulus superimposed on the static plaids as an attentional task. All subjects performed this behavioral task at $>80 \%$ accuracy. Real motion stimuli were identical to the AM stimuli except that they moved horizontally back and forth with smooth motion the speed of which was matched to the AM condition.

Strictly speaking, the presentation of real motion, either surface motion (plaids or random dot kinematograms) or a smooth translocation of a moving patch (our RM stimulus), on a computer screen is also an apparent motion like stimulus, but because the critical fusion frequency of healthy subjects is usually well $<60 \mathrm{~Hz}$, we can safely assume that they indeed perceived smooth real motion during these experiments.

\section{Eye-movement control experiments}

Eye movements were measured with an iViewX high-speed eye tracker (SMI) that allowed for data collection at $240 \mathrm{~Hz}$. Data were collected outside the scanner in eight subjects, using the exact same stimulus protocols as in experiment $2 b$. Data time series were searched for fixation, blinks, and saccadic events with Begaze Software (SMI) and subsequently exported to standard statistical packages (SPSS and Statview).

Analysis of the externally recorded eye-tracking data showed that fixation could be stably held across all conditions, with very rare saccades, and reduced number and duration of blinks, which was similar across conditions. ANOVA did not show any interaction between conditions and fixation patterns.

All experiments were undertaken with the understanding and written consent of each subject following the Guidelines of our local Ethics Committee and the Declaration of Helsinki.

\section{Data analysis}

Data analysis included preprocessing (slice scan time correction, 3D motion correction, and spatial and temporal filtering), coregistration and Tailarach transformation of scans. These steps were performed by using BrainVoyager 2000 and BrainVoyager QX (Brain Innovation B.V., Maastricht, The Netherlands) software. For multiple regression analysis, a general linear model (GLM) was fitted with predictors for each experimental condition. The time courses of individual predictors were obtained by using a linear model of the hemodynamic response (Boynton et al. 1996). The overall model fit was assessed by using an $F$ statistic. Significant differences between the experimental conditions were assessed by using contrast $(t)$ maps. Conjunction analyses of experimental conditions were also performed (using a logical AND criterion for all contrasts). The obtained $P$ values were corrected for multiple comparisons by using a Bonferroni adjustment. Clusters were accepted only as significant when $p_{\text {corr }}<$ 0.001 . The localization of each subject's $\mathrm{hMT}^{+}$was performed by GLM contrasting static conditions with movement conditions.

Baseline percent signal change was calculated for each condition separately based on the average activation level of the immediately preceding fixation period, taking into account the hemodynamic delay. For further analysis, data from subjects and runs were pooled according to stimulus conditions. In experiments $1 b, 2 a$, and $2 b$, ANOVA statistics with Fisher's post hoc comparisons were calculated on the pooled data of the first, second, third, and fourth 8-s epochs of a whole stimulation cycle. For presentation purposes, we show the activation level at data-points corresponding to the acquisition volumes.

\section{R E S U L T S}

\section{Experiment 1-evidence for a net MAE-related motion signal when attention is stably focused on a motion-independent feature}

In experiment $1 a$, we have investigated neural responses to real motion and illusory motion aftereffects when attention is controlled for by motion-independent color and angle tasks. The hypothesis was that if a MAE-related net motion signal exists and is masked by interference with concurrent motion task (as was possibly the case in Huk et al. 2001), this masking should not occur with motion-independent tasks.

We have found strong $\mathrm{hMT}^{+} / \mathrm{V} 5$ responses to stationary plaids in comparison to baseline fixation stimuli (Fig. 3A) in our group data. ANOVA of BOLD responses in these regions of interest showed highly significant differences across motion, stationary, and fixation conditions $(P \ll 0.001$, for all comparisons, with Bonferroni post hoc corrections).

BOLD responses to stationary plaids versus fixation baseline showed a high degree of localization to $\mathrm{hMT}^{+} / \mathrm{V} 5$ (multisubject cluster centers of gravity at $p_{\text {corr }} \ll 0.001: x-48 y$ $-64 z-1$ and $x+47 y-66 z+1)$. To assess whether $\mathrm{hMT}^{+} / \mathrm{V} 5$ was specifically activated by stationary plaids after motion adaptation, we computed a conjunction analysis (which means that only voxels that are significant for all contrasts will survive the statistical thresholding) including the contrast of postadaptation stationary conditions versus the fixation baseline and the contrasts of moving conditions versus fixation baseline. The strict co-localization of all signals (including the MAE-related ones) to the same $\mathrm{hMT}^{+} / \mathrm{V} 5$ clusters suggests that the increased activity measured in $\mathrm{hMT}^{+} / \mathrm{V} 5$ on stimulation with stationary plaids after adaptation to moving plaids is due to a specific net neural motion-related signal (Fig. 3B).

Aside from the expected decay in activity both for postadapting fixation and stationary stimuli (ANOVA, $P<0.0001$, for $\mathrm{hMT}^{+} / \mathrm{V} 5$, bilaterally), there are subtle differences in the temporal pattern of decay (Fig. 3A). Responses to stationary plaids were already higher early on if compared with activation induced by fixation stimuli, which do not induce MAE (ANOVA, Fisher PLSD, $P<0.0001$, for $\mathrm{hMT}^{+} / \mathrm{V} 5$, bilaterally, already within the first 2 volumes, after correction for the hemodynamic delay), showing that high levels of MAE-related activity in the initial period were not signaling a simple decay from the previous period of moving stimulus presentation but rather a genuine initial increase of activity (see experiments $1 b$, $2 a$, and $2 b$ for further evidence). BOLD responses to real surface motion compared with fixation stimuli were also significantly higher already from the beginning of the period and increased thereafter significantly to a stable plateau.

The used attention tasks emphasized either the function of the ventral stream (color task) or the function of the dorsal stream (spatial angle type of task) (Mendes et al. 2005), but the measured activity on motion adaptation was above baseline in $\mathrm{hMT}^{+} / \mathrm{V} 5$ irrespective of the task and regardless of the adaptation condition used (ANOVA, Fisher post hoc, $P \ll 0.01$ for all conditions), and there was no significant difference between the activation patterns observed with the two attentional tasks. It is also worth pointing out that these attentional tasks were 
present throughout both stationary and motion conditions, so no significant signal changes are expected to occur due to attentional fluctuations across conditions (see also Supplementary Fig. S1).

EXPERIMENT 1B. To provide further and definite evidence that specific MAE-related signals can indeed be identified in $\mathrm{hMT}^{+}$, we have performed an independent replication $(n=8)$ of experiment $1 a$ with the additional inclusion of a nonadapting motion (e.g., not eliciting MAEs) control condition (similar to experiments $2 a$ and $2 b$, see Fig. 2). As expected from our
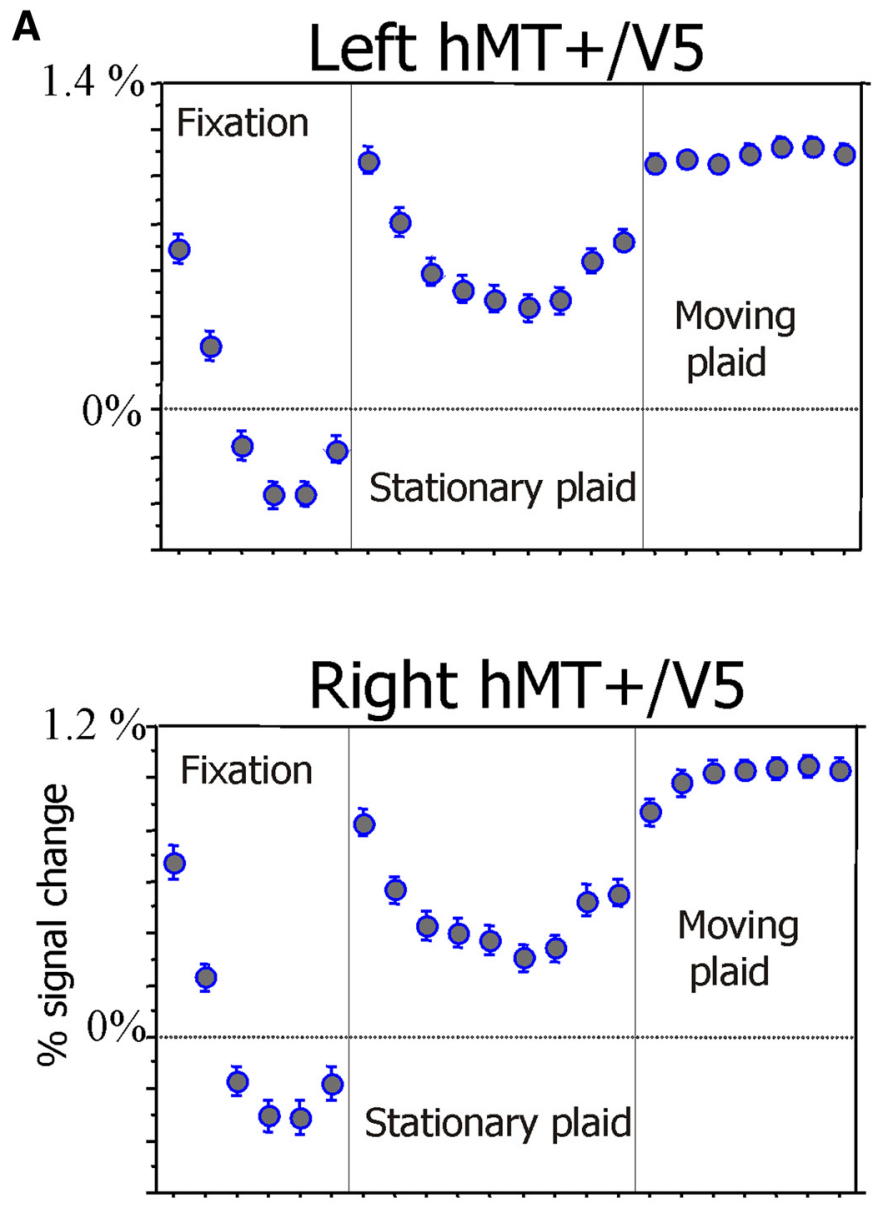

\section{Scans}

B
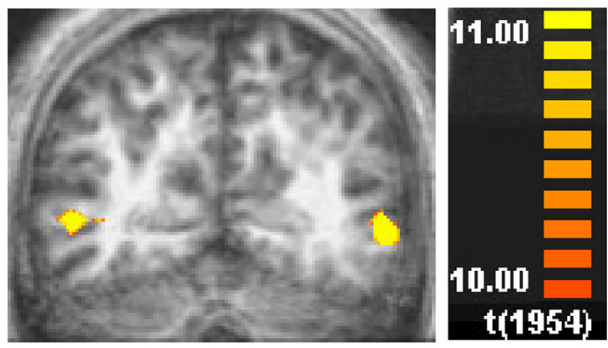

\section{Conjunction analysis (Motion\& Stationary VS Fixation)}

prediction, global $\mathrm{hMT}^{+}$activity persisted at higher levels when either color or angle tasks were superimposed to the static plaid period after the presentation of fixed (adapting, e.g., MAE inducing) plaids as compared with presentation of mixed (nonadapting) plaids (Fig. 4, $P<0.05$ for both comparisons concerning color and angle tasks).

In summary, activity levels in these motion-sensitive areas are increased on plaid motion adaptation, even if disparate motion independent tasks keeping attention stably away from stationary plaid features (color task) or from moving plaid features (spatial comparison task) are used to control for attention. The effect therefore appears to be general across motion independent tasks. Still, it remained an open question whether this would be the case in the presence of selective attention to concurrent orthogonal illusory/real motion features. Experiments $2 a$ and $2 b$ addressed this issue.

\section{Experiment $2 a$ and $2 b-N o$ net MAE-related motion signal when attention is stably focused on a concurrent independent motion feature. Evidence for interference mechanisms between dual motion processes}

In these experiments, we have investigated neural responses to real motion and illusory MAEs, when attention is controlled for by speed-tracking of apparent motion or real motion stimuli. The hypothesis was that if a MAE-related net motion signal exists and is masked by interference with concurrent motion task (as possibly in Huk et al. 2001), this masking should occur with motion-related tasks, regardless of whether motion is apparent or real. Figure 2 illustrates the conditions tested in experiments $2 a$ and $2 b$.

\section{Characterization of responses to superimposed AM}

Before looking at the MAE-related neural signal, we have characterized neural responses during the adaptation period with and without superimposition of apparent or real concurrent motion (AM and RM). This provided a way of assessing possible saturation effects. Figure 5 shows the general BOLDeffect of superimposing AM on adapting or nonadapting plaid movement and subsequent static plaids (yielding MAE for adapting conditions). As expected, global $\mathrm{hMT}^{+}$activity persisted at high levels when AM stimuli were superimposed to the static plaid period after the presentation of mixed (nonadapting) plaids (top left, $P=0.0024$ during the initial 8-s

FIG. 3. A: strong responses to stationary plaids in human motion complex $\left(\mathrm{hMT}^{+} / \mathrm{V} 5\right)$ after plaid motion adaptation (experiment la). Regions of interest (ROIs) were defined by plaid motion contrast, which shows substantial overlap with flow-field mapping (see also Castelo-Branco et al. 2002). The temporal dynamics (per scan) of cortical responses to moving plaids, stationary plaids after adaptation to moving plaids, and fixation baseline stimuli are shown $(n=$ 4 for experiment 1a). Responses to stationary plaids are higher than responses evoked by fixation stimuli already within the 1 st 2 scans, suggesting a response to motion aftereffect (MAE; ANOVA, Fisher PLSD, $P<0.0001$, for $\mathrm{hMT}^{+}$/ V5, bilaterally), and both types of response decay significantly afterward (ANOVA, $P<0.0001$, for $\mathrm{hMT}^{+} / \mathrm{V} 5$, bilaterally). Note that order of conditions is variable and balanced (for details, see METHODS). Error bars in this and subsequent figures depict 1 SE. B: general linear model (GLM) conjunction analysis (criterion being that all contrasts have to be significant for a voxel to be considered positive; for details see text) of blood-oxygenation-level-dependent (BOLD) responses to moving and stationary plaids on motion adaptation shows a quite specific degree of localization to $\mathrm{hMT}^{+}$(multi-subject, multistudy GLM analysis, $n=4$ subjects). Regions are shown at the same statistical threshold level ( $p_{\text {corr }}<0.001$, see color plot). 

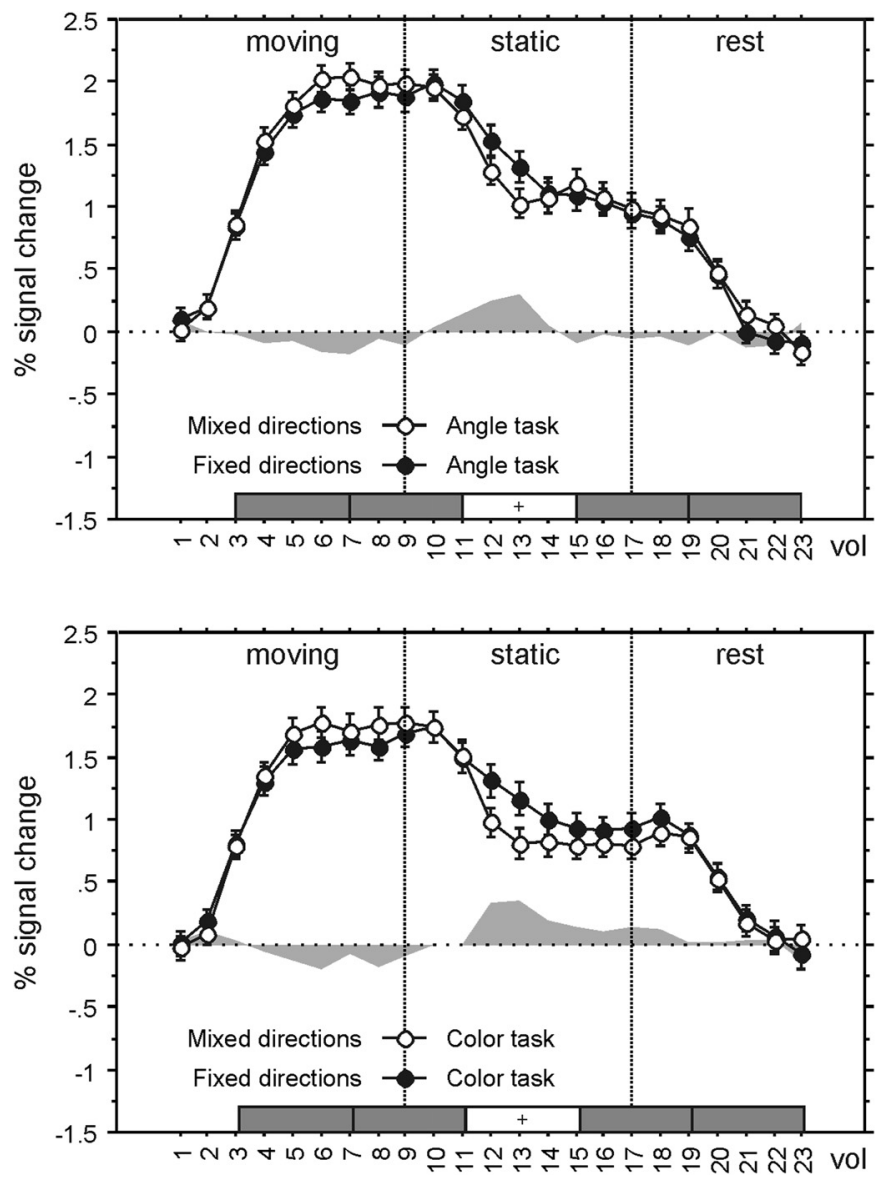

FIG. 4. Independent replication of experiment $1 a$ that includes a mixed (nonadapting, non-MAE inducing) control condition $(n=8$ in this experiment $1 b)$. Global $\mathrm{hMT}^{+}$activity persisted at higher levels when either color or angle tasks were superimposed to the static plaid period after the presentation of fixed (adapting, e.g., MAE inducing) plaids as compared with presentation of mixed (nonadapting) plaids $(P<0.05$ for both color and angle tasks). Time points depict TRs (vol). Bars on the bottom of the graphs represent statistical comparisons for data pooled over 8-s blocks: $\mathbf{}$, nonsignificant difference; $\square$, significant difference $(+: P<0.05$; ++ : $P<0.001$, ANOVA with Fisher's post hoc). Error bars on all plots represent $1 \mathrm{SE}$.

period, and $P<0.0001$ thereafter), signifying a pure AM signal because there can be no, and indeed were no, MAEs perceived after the presentation of mixed plaids. Moreover, a small but significant increase in activity was observed during the motion period compared with a pure plaid-motion activation when AM was overlaid on the moving plaid period (bottom left, $P=$ 0.0294 , bottom white box highlights statistically significant blockwise difference). This shows that $\mathrm{hMT}^{+}$was not reaching saturation with adapting plaid motion.

The investigation of the interaction between AM and MAE revealed a sort of cross-masking: superimposing AM stimuli after adaptation to fixed direction motion eliciting MAEs did not lead to any additive increase in activity (top right, n.s.). This phenomenon was not due to putative saturation because superimposing AM during the presentation of moving fixed plaids lead to a increase in activity during the movement period similar to the one observed with superimposed AM on mixed plaids. Furthermore, a subtle but interesting observation was that this prior superimposition of AM lead to a significant subsequent decay of $\mathrm{hMT}^{+}$activation during the static period in comparison to the presentation of fixed plaids alone (bottom right, $P=0.0018$ ). Because fixed plaid motion was always orthogonal to AM, this may have effectively acted as a mixedlike nonadapting situation with ensuing quicker decline of $\mathrm{hMT}^{+}$activity.

\section{Effects of selective attention to apparent motion on $M A E$-related motion signals}

Given the demonstration that AM differentially modulates nonsaturated $\mathrm{hMT}^{+}$responses during plaid motion presentation (additive effect), we have finally focused on response properties during the ensuing stationary plaid presentation epoch (which revealed a masking effect of concurrent motion on the MAE signal).

If AM is absent during the static period (Fig. 6, experiment $2 a$ ), a significant difference in BOLD response can be observed during the static and rest periods, corresponding to stronger responses (and presence of MAEs) after exposure to fixeddirection moving surfaces in comparison to mixed ones (top $P<0.0001$ in first two blocks and $P=0.0009$ in the last one). However, this difference disappears if AM has been overlaid during the static periods (middle, see initial decrease of gray shaded area as compared with the top panel), showing the above-mentioned masking effect (similar to the one observed by Huk et al. 2001).

Interestingly, adding AM both during fixed motion and the ensuing static period did not change responses during the latter (bottom). Although there is a steeper initial increase in activity to significantly higher levels $(P=0.025)$ if AM was superimposed on fixed direction plaid motion (again proving that plaid motion does not saturate $\mathrm{hMT}^{+}$responses per se), the subsequent activation levels elicited by static displays overlaid with AM do not differ significantly. Nonetheless, there is a subtle but clear selective decrease of activation shortly after plaid motion was stopped in the condition where AM has already been present during plaid movement.

\section{Comparison of the modulation effects caused by apparent and real motion tasks during the MAE test period}

In this experiment (experiment 2b), performed in eight additional subjects at $3 \mathrm{~T}$, we have fully replicated the AM experiment and have concomitantly included a new condition, consisting of overlaid real motion using similar stimuli as in the AM condition, on top of the MAE period, (for details see METHODS). We have observed, as expected, a significant difference $(P<0.05)$ during the static test period after adaptation to fixed- versus mixed-direction moving plaids (Fig. 7, top). However, this effect is abolished when subjects have to selectively attend either overlaid AM or RM during the test period (middle and bottom, respectively), thereby providing strong additional evidence for a dual motion interference effect.

\section{I S C U S S I O N}

We have shown here that a net $\mathrm{hMT}^{+}$response is present when a MAE is perceived and is detectable provided selective attention is controlled for using a task that does not interfere with motion processing per se. When attention is controlled for with concomitant motion tasks, this may lead to suppression of the net response due to perceptual interference between motion features. We do believe that our results do reconcile the 

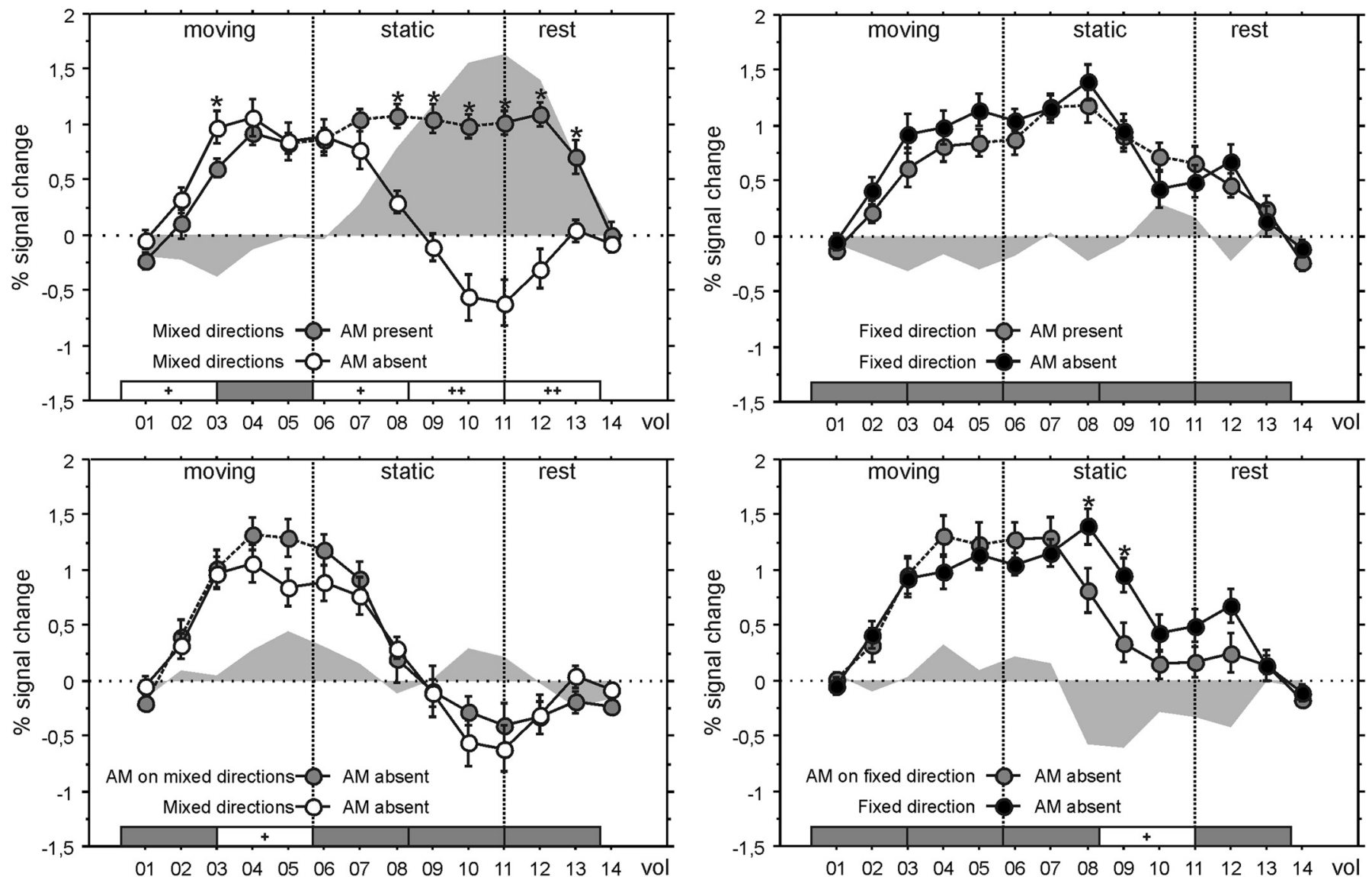

FIG. 5. BOLD response curves in $\mathrm{hMT}^{+} / \mathrm{V} 5$ and their dependence on the presence of apparent motion (AM). Data from individual ROIs averaged across subjects and hemispheres (experiment $2 a$ ). Top left: mixed (nonadapting) motion vs. mixed motion with AM overlaid on static plaid. Bottom left: mixed motion vs. mixed motion with AM overlaid during plaid motion. Top right: fixed (adapting) motion vs. fixed motion with AM overlaid on static plaid. Bottom right: fixed motion vs. fixed motion with AM overlaid during plaid motion. *, significance for volume-wise comparisons. Time points depict TRs (vol). Bars on the bottom of the graphs represent statistical comparisons for data pooled over 8-s blocks: $\mathbf{m}$, nonsignificant difference; $\square$, significant difference $(+: P<0.05 ;++: P<$ 0.001, ANOVA with Fisher's post hoc). Error bars on all plots represent 1 SE.

apparently contradictory results of Tootell et al. (1995) and Huk et al. (2001); by showing that selective attention can suppress motion-adaptation-related activity in $\mathrm{hMT}^{+}$if and only if it is focused on concomitant motion features, regardless of whether they are apparent or real. In other words, perceptual interference/masking does specifically occur if another motion task or stimulus is added even if it is illusory.

Although our results replicate the findings of Huk et al. (2001); they also provide evidence for the existence of a genuine motion signal in $\mathrm{hMT}^{+}$, related to MAE, which is distinctly modulated by the type of difficulty-matched attention task used. The genuine MAE-related signal in $\mathrm{hMT}^{+}$can therefore be separated from the attentional mechanisms by which it is modulated (Berman and Colby 2002; Chaudhuri 1990; Georgiades and Harris 2000, 2002; Rees et al. 1997).

Although the main goal of this study was to investigate how selective attention processes modulate $\mathrm{hMT}^{+}$processing evoked by motion aftereffects, we have also analyzed the differential type of interaction between illusory (AM) and real motion signals prior to the adaptation phase. This also allowed ruling out saturation effects. We could show that net MAErelated activity can be identified in area $\mathrm{hMT}^{+}$under sustained selective attention unrelated to motion perception, regardless of whether we used a color task or a spatial angle comparison task. When selective attention was focused on a real or appar- ent motion task, the MAE-related processing, as probed by fMRI, was masked, even if subjects reported perceiving MAEs. Moreover, we identified additive interactions between plaid movement and AM/RM during the adaptation phase (excluding saturation), and nonadditive (masking) interactions during the MAE test period.

\section{Selective attention and MAE processing}

We have proved that distinct types of selective featural attention mechanisms differentially modulate motion aftereffect-evoked activity in $\mathrm{hMT}^{+}$by showing that motion-unrelated tasks did not cancel MAE-related activations, regardless of whether attention was focused on something other than the moving stimulus (color task) or when its static features still had to be attended to (angle comparison task).

It is worth pointing out that the locus of spatial attention is distinct in these tasks. However, results were similar for the color and angle tasks (see also Supplementary Fig. S1); this shows that these results generalize across distinct conditions. Most importantly, these differences were shown to hold true under precisely matched conditions, such as when comparing $\mathrm{hMT}^{+}$activity after adaptation to fixed motion (MAE inducing) versus nonadapting (mixed motion) conditions. 

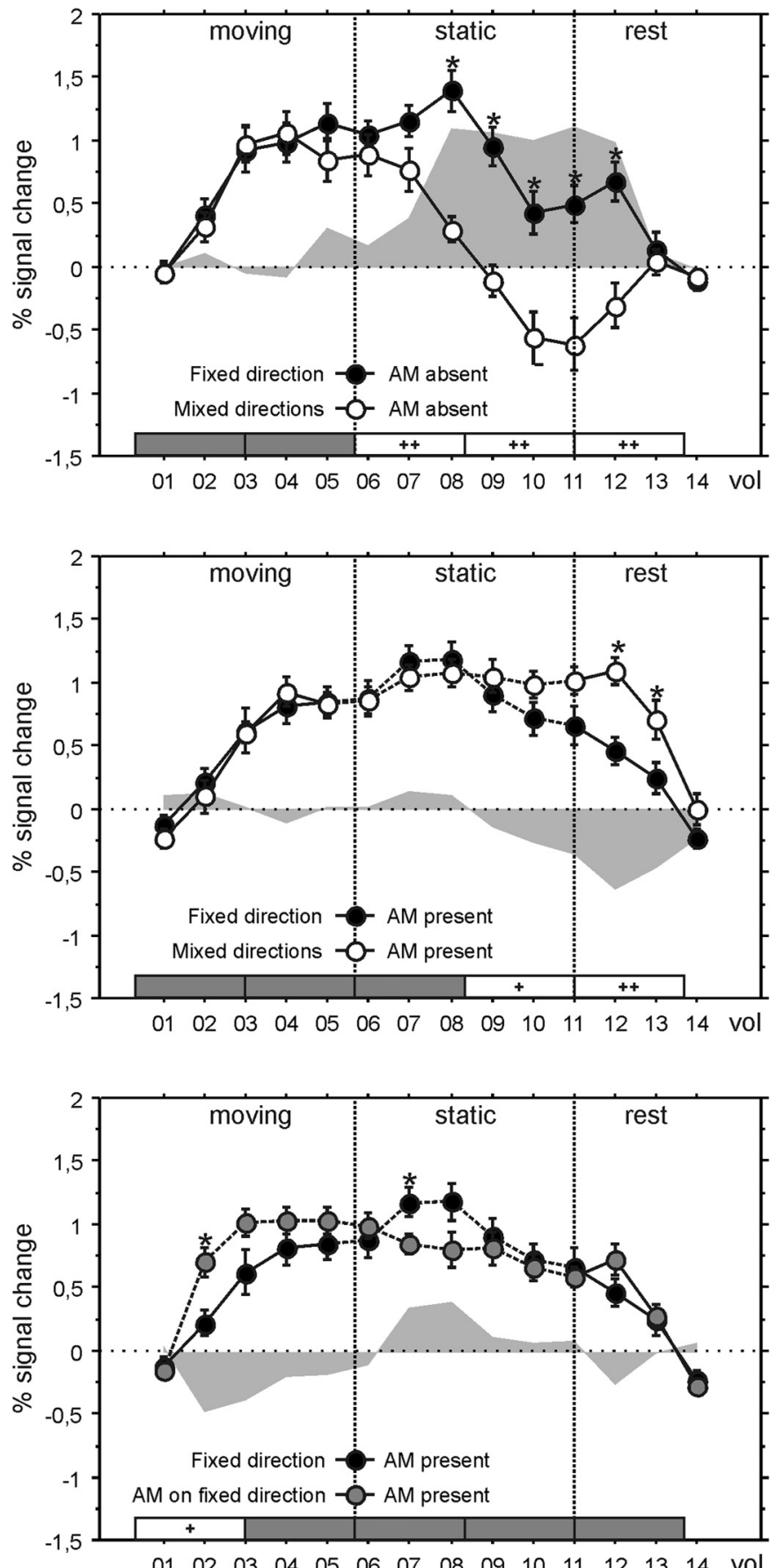

FIG. 6. BOLD response curves in $\mathrm{hMT}^{+} / \mathrm{V} 5$ and modulation of MAEs by presence or absence of AM. Data from individual ROIs averaged across subjects $(n=4)$ and hemispheres $(n=8)$. Top: fixed vs. mixed motion. Middle: fixed vs. mixed motion with AM overlaid during static plaid. Bottom: fixed motion with AM overlaid on static plaid vs. fixed motion with AM overlaid on both plaid motion and static period. *, significance for volume-wise comparisons. Time points depict TRs (vol). Bars on the bottom of the graphs represent statistical comparisons for data pooled over 8-s blocks: घ, nonsignificant difference; $\square$, significant difference $(+: P<0.05 ;++: P<$ 0.001, ANOVA with Fisher's post hoc). Error bars on all plots represent $1 \mathrm{SE}$.

On the other hand, the net increase of activity in $\mathrm{hMT}^{+}$that is evoked by motion adaptation underlying MAEs (Corbetta et al. 1990; Culham et al. 1999; He et al. 1998; Tootell et al. 1995) was masked when we imposed selective attention to AM or RM. These findings suggest that selective attention to concurrent motion features leads to interference that masks the MAE-evoked signal. These results are consistent with and extend the findings of Huk et al. 2001 using a task in which a slight physical motion was added to half of the MAE test stimulus and requiring the subjects to perform a speed comparison task. Under these conditions, they found no net increase in $\mathrm{hMT}^{+}$activity in the presence of a MAE condition


FIG. 7. BOLD response curves in $\mathrm{hMT}^{+} / \mathrm{V} 5$ and comparison of modulation of MAEs by the presence of selective attention to RM or AM as required by a speed judgment task (experiment $2 b, n=8$ ). Time points depict TRs (vol). Bars on the bottom of the graphs represent statistical comparisons for data

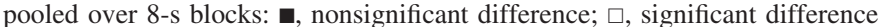
$(+: P<0.05 ;++: P<0.001$, ANOVA with Fisher's post hoc). Error bars on all plots represent $1 \mathrm{SE}$. 
(adaptation under constant direction of motion) as compared with the no-MAE condition (adaptation under mixed directions, which in general does not elicit a MAE).

The discrepancy between the activation patterns observed with different attention tasks further emphasize the special case of selective attention tasks that focus directly on motion perception and is in agreement with the previous literature (Alais and Blake 1999; Culham et al. 2000; Lankheet and Verstraten 1995; Mukai and Watanabe 2001; Shulman 1993; Sohn et al. 2001).

Even though specific superposition of the AM/RM speed tracking tasks during the static test phase substantially reduced the BOLD difference between adapting fixed and nonadapting mixed conditions, a finding that was not observed with the motion-unrelated tasks, our participants reported MAEs only on fixed conditions. This finding suggests that the interaction across perceptual and attentional networks is distinct within (all motion-related) and across feature (motion versus non motion-related) domains.

Because color-matching is associated with ventral-stream function, one cannot expect a very strong interaction within common processing pathways, but interestingly, angle-matching does not either cause interference, in spite of its relatedness to dorsal-stream function, as is the case for the motion tasks showing a marked interaction with MAE (see Castelo-Branco et al. 2007, 2009 for evidence that these types of tasks are specific to the dorsal stream). Our observations, namely suppression of MAE-evoked increase of activity using attention to AM/RM motion stimuli showed that the specific type of featural attention is critically involved in the modulation of net activity in $\mathrm{hMT}^{+}$. In other words, attention to motion provides a qualitatively distinct masking effect, while task difficulty is not a relevant explanatory variable because we found distinct modulation patterns even though difficulty levels were adjusted across our temporally sustained tasks.

We were able to generate control conditions for selective attention without disrupting motion adaptation phenomena per se. Indeed, superposition of orthogonal AM during plaid motion produced a clear-cut increase in the BOLD response of $\mathrm{hMT}^{+}$comparable to a modulation pattern of an additional moving surface (Castelo-Branco et al. 2002), and we were able to show that $\mathrm{hMT}^{+}$responses were not saturated by the motion conditions.

\section{Summary}

We conclude that a genuine neural substrate of illusory motion aftereffect perception can be identified in conditions requiring selective attention to concomitant nonmotion features and is masked by concurrent apparent/real motion tasks. In other words, $\mathrm{hMT}^{+}$sensitivity to MAE can be differentially modulated by selective attention to various nonmotion and motion features. Finally, superimposed real and apparent motion may lead to additive adaptation effects without saturation of $\mathrm{hMT}^{+}$responses, whereas the interaction of simultaneous apparent motion and MAE reflects interference effects.

\section{A C K N O W LEDGMENTS}

We acknowledge the support of Rede Nacional de Imagiologia Funcional Cerebral (the national imaging network of Portugal). Experiments 2 and 3 were performed using Cogent 2000 developed by the Cogent 2000 team at the Functional Imaging Laboratory and the Institute of Cognitive Neuroscience and Cogent Graphics developed by J. Romaya at the Laboratory of Neurobiology at the Wellcome Department of Imaging Neuroscience.

\section{G R A N T S}

This work was supported by Foundation for Science and Technology Portugal Grants POCI/SAU/NEU/60281/2004, PTDC/SAU/NEU/68483/2006 and PTDC/PSI/67381/2006 to M. Castelo-Branco and SFRH/BD/13344/2003 to L. R. Kozak, funding from the Programa de Estimulo à Investigação and Ageing Program of the Calouste Gulbenkian Foundation to L. R. Kozak and M. Castelo-Branco, and by a Bial Foundation Grant to M. Castelo-Branco.

\section{REFERENCES}

Alais D, Blake R. Neural strength of visual attention gauged by motion adaptation. Nat Neurosci 2: 1015-1018, 1999.

Anstis S, Verstraten FAJ, Mather G. The motion aftereffect. Trends Cogn Sci 2: $111-117,1998$.

Beauchamp MS, Cox RW, DeYoe EA. Graded effects of spatial and featural attention on human area MT and associated motion processing areas. J Neurophysiol 78: 516-520, 1997.

Berman RA, Colby CL. Auditory and visual attention modulate motion processing in area MT+. Brain Res Cogn Brain Res 14: 64-74, 2002.

Boynton GM, Engel SA, Glover GH, Heeger DJ. Linear systems analysis of functional magnetic resonance imaging in human V1. J Neurosci 16: 4207-4221, 1996.

Braddick OJ, O'Brien JM, Wattam-Bell J, Atkinson J, Turner R. Form and motion coherence activate independent, but not dorsal/ventral segregated, networks in the human brain. Curr Biol 10: 731-734, 2000.

Buchel C, Josephs O, Rees G, Turner R, Frith CD, Friston KJ. The functional anatomy of attention to visual motion. A functional MRI study. Brain 121:1281-1294, 1998.

Castelo-Branco M, Formisano E, Backes W, Zanella F, Neuenschwander S, Singer W, Goebel R. Activity patterns in human motion-sensitive areas depend on the interpretation of global motion. Proc Natl Acad Sci USA 99: 13914-13919, 2002.

Castelo-Branco M, Mendes M, Sebastiao AR, Reis A, Soares M, Saraiva J, Bernardes R, Flores R, Perez-Jurado L, Silva E. Visual phenotype in Williams-Beuren syndrome challenges magnocellular theories explaining human neurodevelopmental visual cortical disorders. J Clin Invest 117: 3720-3729, 2007.

Castelo-Branco M, Mendes M, Silva MF, Januario C, Machado E, Pinto A, Figueiredo P, Freire A. Specific retinotopically based magnocellular impairment in a patient with medial visual dorsal stream damage. Neuropsychologia 44: 238-253, 2006.

Castelo-Branco M, Mendes M, Silva F, Massano J, Januario G, Januario C, Freire A. Motion integration deficits are independent of magnocellular impairment in Parkinson's disease. Neuropsychologia 47: 314-20, 2009.

Chaudhuri A. Modulation of the motion aftereffect by selective attention. Nature 344: 60-62, 1990.

Corbetta M, Miezin FM, Dobmeyer S, Shulman GL, Petersen SE. Attentional modulation of neural processing of shape, color, and velocity in humans. Science 248: 1556-1559, 1990.

Culham JC, Dukelow SP, Vilis T, Hassard FA, Gati JS, Menon RS, Goodale MA. Recovery of fMRI activation in motion area MT following storage of the motion aftereffect. J Neurophysiol 81: 388-393, 1999.

Culham JC, Verstraten FA, Ashida H, Cavanagh P. Independent aftereffects of attention and motion. Neuron 28: 607-615, 2000.

Georgiades MS, Harris JP. The spatial spread of attentional modulation of the motion aftereffect. Perception 29: 1185-1201, 2000.

Georgiades MS, Harris JP. Effects of attentional modulation of a stationary surround in adaptation to motion. Perception 31: 393-408, 2002.

Goebel R, Khorram-Sefat D, Muckli L, Hacker H, Singer W. The constructive nature of vision: direct evidence from functional magnetic resonance imaging studies of apparent motion and motion imagery. Eur J Neurosci 10: 1563-1573, 1998.

Hautzel H, Taylor JG, Krause BJ, Schmitz N, Tellmann L, Ziemons K, Shah NJ, Herzog H, Muller-Gartner HW. The motion aftereffect: more than area V5/MT? Evidence from 15O-butanol PET studies. Brain Res 892: 281-292, 2001.

He S, Cohen ER, Hu X. Close correlation between activity in brain area MT/V5 and the perception of a visual motion aftereffect. Curr Biol 8: 1215-1218, 1998.

Huk AC, Heeger DJ. Pattern-motion responses in human visual cortex. Nat Neurosci 5: 72-75, 2002. 
Huk AC, Ress D, Heeger DJ. Neuronal basis of the motion aftereffect reconsidered. Neuron 32: 161-172, 2001.

Kastner S, Pinsk MA, De Weerd P, Desimone R, Ungerleider LG. Increased activity in human visual cortex during directed attention in the absence of visual stimulation. Neuron 22: 751-761, 1999.

Kozak LR, Castelo-Branco M. Peripheral influences on motion integration in foveal vision are modulated by central local ambiguity and center-surround congruence. Invest Ophthalmol Vis Sci 50: 980-988, 2009.

Lankheet MJ, Verstraten FA. Attentional modulation of adaptation to two-component transparent motion. Vision Res 35: 1401-1412, 1995.

Liu T, Slotnick SD, Yantis S. Human $\mathrm{MT}^{+}$mediates perceptual filling-in during apparent motion. Neuroimage 21: 1772-1780, 2004.

Mendes M, Silva F, Simões L, Jorge M, Saraiva J, Castelo-Branco M. Visual magnocellular and structure from motion perceptual deficits in a neurodevelopmental model of dorsal stream function. Cogn Brain Res 25: 788-98, 2005

Mikami A, Newsome WT, Wurtz RH. Motion selectivity in macaque visual cortex. I. Mechanisms of direction and speed selectivity in extrastriate area MT. J Neurophysiol 55: 1308-1327, 1986a.

Mikami A, Newsome WT, Wurtz RH. Motion selectivity in macaque visual cortex. II. Spatiotemporal range of directional interactions in MT and V1. J Neurophysiol 55: 1328-1339, 1986b.

Muckli L, Kriegeskorte N, Lanfermann H, Zanella FE, Singer W, Goebel R. Apparent motion: event-related functional magnetic resonance imaging of perceptual switches and states (Rapid communication). J Neurosci 22: RC219, 2002.

Mukai I, Watanabe T. Differential effect of attention to translation and expansion on motion aftereffects (MAE). Vision Res 41: 1107-1117, 2001

Newsome WT, Mikami A, Wurtz RH. Motion selectivity in macaque visual cortex. III. Psychophysics and physiology of apparent motion. J Neurophysiol 55: 1340-1351, 1986.
O'Craven KM, Rosen BR, Kwong KK, Treisman A, Savoy RL. Voluntary attention modulates fMRI activity in human MT-MST. Neuron 18: 591-598, 1997.

Rees G, Frith CD, Lavie N. Modulating irrelevant motion perception by varying attentional load in an unrelated task. Science 278: 1616-1619, 1997.

Seidemann E, Newsome WT. Effect of spatial attention on the responses of area MT neurons. J Neurophysiol 81: 1783-1794, 1999.

Shulman GL. Attentional effects of adaptation of rotary motion in the plane. Perception 22: 947-961, 1993.

Sohn, Vidnyanszky, Blaser, Papathomas. Attention to one component of bivectorial transparent motion strongly inhibits the processing of the unattended component. J Vision 1: 85-85, 2001.

Sunaert S, Van Hecke P, Marchal G, Orban GA. Motion-responsive regions of the human brain. Exp Brain Res 127: 355-370, 1999.

Taylor JG, Schmitz N, Ziemons K, Grosse-Ruyken ML, Gruber O, Mueller-Gaertner HW, Shah NJ. The network of brain areas involved in the motion aftereffect. Neuroimage 11: 257-270, 2000.

Tootell RB, Reppas JB, Dale AM, Look RB, Sereno MI, Malach R, Brady TJ, Rosen BR. Visual motion aftereffect in human cortical area MT revealed by functional magnetic resonance imaging. Nature 375: 139-141, 1995.

Tootell RB, Taylor JB. Anatomical evidence for MT and additional cortical visual areas in humans. Cereb Cortex 5: 39-55, 1995.

Treue S, Martinez Trujillo JC. Feature-based attention influences motion processing gain in macaque visual cortex. Nature 399: 575-579, 1999.

Verstraten FAJ, van der Smagt MJ, van de Grind WA. Aftereffect of high-speed motion. Perception 27: 1055-1066, 1998.

Watson JD, Myers R, Frackowiak RS, Hajnal JV, Woods RP, Mazziotta JC, Shipp S, Zeki S. Area V5 of the human brain: evidence from a combined study using positron emission tomography and magnetic resonance imaging. Cereb Cortex 3: 79-94, 1993. 